\title{
La portée structurante de l'interdit. Eléments pour une « clinique » de l'autorité
}

\section{Dominique Ginet}

\section{OpenEdition}

1 Journals

\section{Édition électronique}

URL : http://journals.openedition.org/trema/493

DOI : 10.4000/trema.493

ISSN : 2107-0997

\section{Éditeur}

Faculté d'Éducation de l'université de Montpellier

\section{Édition imprimée}

Date de publication : 1 mars 2007

Pagination : 47-55

ISSN : 1167-315X

\section{Référence électronique}

Dominique Ginet, «La portée structurante de l'interdit. Eléments pour une « clinique » de l'autorité », Tréma [En ligne], 27 | 2007, mis en ligne le 03 mars 2010, consulté le 19 avril 2019. URL : http:// journals.openedition.org/trema/493 ; DOI : 10.4000/trema.493

Ce document a été généré automatiquement le 19 avril 2019

Trema 


\title{
La portée structurante de l'interdit. Eléments pour une « clinique » de l'autorité
}

\author{
Dominique Ginet
}

\section{Introduction}

1 Cette "crise » actuelle de l'autorité, l'on ne s'étonnera pas d'en voir apparaître les symptômes essentiellement dans les espaces de rencontre entre les générations, au sein des familles, d'abord, mais surtout dans l'Ecole. Et, en dépit de son aspect protéiforme, une telle crise désigne ce qui est de l'ordre d'un refus, d'une récusation, voire d'une invalidation de l'autorité de l'adulte, de l'aîné, de l'enseignant ... Ce qui serait aujourd'hui rejeté, c'est cette parole qui fixe une limite. Ce qui paraît mis à mal, c'est le «cadre » de travail de l'Ecole. Ce qui est subverti, c'est la mise en rôle respective qu'implique l'organisation scolaire. Ce qui semble voué désormais à l'insignifiance, ce serait le sens même de l'Ecole, la transmission du Savoir, l'acquisition des connaissances.

\section{II. « Jongler avec les interdits »?}

2 Un exemple récent? Cette rencontre avec l'équipe pédagogique d'une classe de 4e, dans un collège de la banlieue lyonnaise. Des enseignants qui semblent soulagés d'être - enfin entendus par un tiers, externe à l'établissement, et qui me confient : « Dès le départ, ça a été difficile avec cette classe, c'est parti très vite à la dérive... Ils ont des comportements incompatibles avec un cours, ils sont violents, dispersés, bruyants, n'arrêtent pas de se lever ; sur une heure, on ne peut faire que dix minutes de cours normal ... Sur eux, nous n'avons pas de prise, nous n'avons aucune ficelle à tirer! Individuellement, ils sont charmants, dès qu'ils sont en groupe, tout est annulé ... Ce sont des gouffres d'ignorance, et pourtant, ils sabotent tout. Nous nous sentons harcelés, ça nous gâche la vie ; ils jonglent avec les interdits ${ }^{1}$. Face à eux, c'est comme si nous, les profs, nous n'existions pas. Et ils 
montent des cabales contre nous... Et leurs parents, quant à eux, sont complètement démunis... ».

3 J'entends donc parler d'élèves - des adolescents - qui, non seulement ne semblent rien attendre de l'Ecole, mais encore qui «jouent » à l'attaquer, à la réduire à l'impuissance, voire à la tourner en dérision; des élèves qui, selon la formule de Jacques LÉVINE, sont en situation de totale «dépactisation» d'avec le système scolaire ${ }^{2}$. Mais ce que j'entends surtout, c'est le désarroi de professionnels de l'éducation scolaire, confrontés à l'inanité de leurs efforts ; ils se sentent disqualifiés dans leur rôle et leur identité d'enseignants, réduits à une position d'inefficacité par leurs propres élèves et engagés, par cette situation, dans un vécu particulièrement douloureux, qu'ils s'attachent à me faire ressentir: "C'est terrible d'en venir à détester ses élèves ... Ils nous désespèrent vraiment. Par rapport à eux, ce que je ressens, c'est de la tristesse ... Tout ce que nous leur proposons, ça ne les intéresse pas, ne les concerne pas ... ». Et, au delà de la multiplication des demandes de conseils de discipline, dont ils conviennent bien que celles - ci ne constituent pas une vraie solution, ils admettront trouver un certain réconfort dans le renforcement de leurs liens au sein de l'équipe pédagogique, dans la mise en place, quasi spontanément, d'un co - étayage réciproque.

Qu'une telle situation ne soit ni isolée, ni exceptionnelle, c'est ce dont tous les acteurs de l'éducation, familiale ou scolaire, conviendront sans peine. Mais c'est bien plutôt en direction d'une mise en intelligibilité de ce type de situation - symptôme qu'il est nécessaire d'oeuvrer, au moment même où les praticiens s'avouent désorientés, voire sidérés, et parfois résignés devant de tels phénomènes... Ne devons - nous pas reconnaître que les travaux d'un Pierre LEGENDRE ${ }^{3}$ sur la transmission intergénérationnelle nous fournissent, avec une hauteur de vue suffisante, le cadre adéquat pour penser cette problématique? Si, de tout temps, la responsabilité incontournable de l'humanité est bien de transmettre aux générations des plus jeunes - et par une voie extra - biologique, qui est donc forcément celle du langage - un "objet biface ", qui comporte d'un côté le savoir, les connaissances, et de l'autre la Loi, la limite, les interdits, n'est - ce pas alors précisément cette transmission qui se trouve de facto mise en panne, et comme enrayée par les attitudes de ces jeunes?

Une telle occurrence apparaît dès lors comme radicalement neuve dans le champ des pratiques éducatives: ce qui se donnerait à entendre, à travers la diversité des symptômes de la crise actuelle, serait en réalité la dimension d'une triple récusation: d'une part, celle des modalités, instituées par l'Ecole, de la transmission - mais l'on peut penser que ce n'est pas là un aspect bien nouveau, l'histoire de l'éducation nous en montrerait plutôt le caractère itératif -; d'autre part, celle de l'agent de cette transmission, l'enseignant, qui est en position de médiateur entre l'élève et l'objet de la transmission; évalué incompétent, il est perçu comme illégitime. Récusation plus nouvelle, enfin, que celle de l'objet même de la transmission, dans sa dualité : nous aurions affaire ici, simultanément, à une dévaluation tant du savoir (" tout ce que nous leur proposons, ça ne les intéresse pas ») que de la Loi et de son énonciation à travers l'interdit (« ils jonglent avec les interdits ... »).

\section{D'un discrédit social de l'interdit ?}

6 Les termes selon lesquels S. FREUD, en 1932, évoque la dimension de l'interdit dans l'éducation sont bien connus : «L'éducation a donc à chercher sa voie entre le Scylla du 
laisser - faire et le Charybde du refuser ... Il s'agira de décider jusqu'où on a le droit d'interdire, en quels temps et par quels moyens $»^{4}$. Chercher sa voie ? C'est - à - dire trouver un équilibre, toujours précaire, toujours incertain, avec, à chaque instant, le risque de se trouver, en tant qu'éducateur, dans une position d'excès, le laxisme d'un côté, l'autoritarisme de l'autre. Des excès que FREUD ne se prive pas de stigmatiser, lorsqu'il écrit dans le même texte: «La moindre réflexion enseigne que l'éducation, jusqu'à présent, a très mal accompli sa tâche et a infligé de grands dommages aux enfants » (o.c., p. 234). Cependant, le point sur lequel les vues de FREUD rejoignent de façon quasi - prophétique certains aspects de la crise actuelle de l'autorité éducative, concerne son évocation - fictive, à ses yeux - d'une éducation sans limites, sans interdits : "L'enfant doit apprendre la domination sur les pulsions. Lui donner la liberté de suivre sans restriction toutes ses impulsions est impossible. Ce serait une expérimentation très instructive pour les psychologues d'enfants, mais en ce cas les parents ne pourraient pas vivre, et les enfants eux - mêmes subiraient un grave dommage qui se manifesterait pour une part immédiatement, pour une autre part dans les années ultérieures. » (Ibid., p. 233). Nous observerons seulement qu'il y a là, présente dans la pensée de FREUD, une forte intuition de la portée psychiquement structurante de l'interdit.

Or, ce que FREUD évoque à titre " expérimental », n'est-ce pas précisément ce à quoi nous sommes présentement confrontés, non point seulement d'une façon individuelle, mais bien plutôt sur un plan sociétal ? Mais alors, s'il en va bien ainsi, comment construire une intelligibilité d'une pareille évolution? Sans entrer dans le détail de l'histoire des pratiques éducatives, ne peut-on suggérer que le modèle de l'éducation " traditionnelle ", qui stipulait une mise en rôle rigide des partenaires de la transaction éducative, imposait à l'éduqué soumission et docilité (à défaut d'obéissance), et préférait souvent la jouissance de l'autoritarisme à l'ascèse de l'autorité, a été peu à peu soumis à une lente érosion. La violence de ses abus eux - mêmes n'a pu que contribuer à son discrédit, en mettant en question sa pérennité ${ }^{5}$.

8 Sous cet angle, on ne manquera pas de remarquer que, dès 1938, Jacques LACAN, s'attachant à comprendre les effets psychiques de l'évolution de la famille, diagnostique la désuétude du pôle paternel et l'affaiblissement de l'autorité, dont il est traditionnellement le garant: "Mais un grand nombre d'effets psychologiques nous semblent relever d'un déclin social de l'imago paternelle. Déclin conditionné par le retour sur l'individu d'effets extrêmes du progrès social, déclin qui se marque surtout de nos jours dans les collectivités les plus éprouvées par ses effets : concentration économique, catastrophes politiques... Ce déclin constitue une crise psychologique. Peut - être est - ce à cette crise qu'il faut rapporter l'apparition de la psychanalyse elle - même $»^{6}$.

9 Ce que nous appelons le discrédit « social » de l'interdit, et qui - à nos yeux - n'engage pas de façon mécanique la dimension «symbolique» de celui - ci, ne trouve - $t$ - il pas son acmé dans les thématiques émergeantes des «événements » de Mai 68 ? Le slogan : « il est interdit d'interdire", qui est encore dans tous les esprits, visait-il autre chose qu'à récuser l'insupportable d'une parole répressive - une répression qui ne porterait rien moins que sur le Désir lui - même - au prix du déni de sa fonction d'organisation psychique, lequel renvoie de la part de l'adulte à ce que Jacques LÉVINE appelle, quant à lui, un "déni de responsabilité ». Nous ne pouvons entrer ici dans le rappel des idéologisations auxquelles ont donné naissance, et de manière proliférante, de telles positions psychiques, dont le point commun est une sorte de révérence fétichique quant à la toute - puissance de l'enfant et le souci constant de lui éviter toute frustration. Ce qui 
nous importe bien plutôt, c'est d'évoquer le phénomène massif de " déparentalisation »", entrainé par le refus ou l'impossibilité de tant d'adultes, enseignants ou parents, de «tenir » une position d'autorité à l'égard de plus jeunes. Les années 70 se sont ainsi caractérisées par des cohortes de jeunes adultes, raidis dans des contre - attitudes réactionnelles par rapport à leur propre éducation, et convaincus d'une seule chose, c'est qu'il ne fallait pas faire comme leurs parents, comme leurs profs ...

\section{L'apport de Françoise Dolto : les « castrations »}

10 L'immense mérite de F. DOLTO, qui a souvent répété que sa vocation était de devenir «médecin d'éducation », a été d'ouvrir résolument la voie d'une approche « clinique » de l'éducation. Là où $\mathrm{S}$. FREUD nous livre une description presque entomologique de la psychogenèse libidinale, à travers les «stades » du développement psycho - affectif - et il était bien entendu inévitable d'en commencer par là - DOLTO va nous faire entendre la nécessité de dialectiser ce point de vue, en intégrant l'univers complexe des influences éducatives qui pèsent sur l'enfant. Ainsi peut-elle observer: "Dans la dynamique familiale, c'est beaucoup plus l'inconscient qui est l'agent de l'éducation, réussie ou non, qu'un savoir pédagogique appris $»^{8}$. Et, pour nous limiter à l'objectif de ces remarques, à savoir éclairer la valeur psychiquement structurante de l'interdit, nous nous appuierons essentiellement sur son ouvrage de 1984, "L'image inconsciente du corps", qui récapitule de multiples publications antérieures, plus succinctes. L'intérêt de son apport consiste à resignifier la notion d'interdit par le concept de "castration », lequel n'a plus le sens de mutilation génitale qu'il avait dans l'œuvre de FREUD et de ses collègues. Tout au contraire, pour DOLTO, la castration est un interdit « symboligène », c'est - à - dire qui fait entrer davantage l'enfant dans l'ordre symbolique, qui le fait parler et grandir, donc.

\section{IV.1. L' « inter-dit »}

11 C'est Denis VASSE, un élève de Françoise DOLTO, qui propose cette modification d'écriture du mot d'interdit afin de mettre en exergue cette dimension du «dire», de l'énonciation de ce moment de la Loi : l'interdit est véritablement une Parole adressée par un aîné à un enfant, non pas pour le frustrer, mais pour l'aider à grandir : «La zone érogène ne peut être introduite au langage de la parole qu'après avoir été privée totalement de l'objet spécifique par lequel elle avait été initiée à la communication érotique. Et cela n'est possible que si le même objet total (la mère) vocalise les phonèmes de mots qui spécifient cette zone érogène: "Le sein de ta mère t'est défendu maintenant » ... C'est la parole qui, du fait de la fonction symbolique, entraîne mutation de niveau du désir. » (o.c. p. 64).

Insistons sur l'immense portée de ce qu'il y a lieu de lire dans ces lignes et qui constitue l'originalité courageuse de l'apport de F. DOLTO : l'interdit doit porter sur l'objet de la pulsion et non sur la pulsion elle - même! Il est très exactement désigné par DOLTO comme ce trait séparateur qui s'insère entre la poussée pulsionnelle et l'objet de satisfaction de celle - ci : renouvellement considérable de notre façon de concevoir l'éducation, au terme de quelques vingt siècles de domination d'un modèle "judéo chrétien ", qui n'a cesser de diaboliser la pulsion, pour la mieux répudier ! F. DOLTO indique simplement que la pulsion, en tant qu'elle fait partie de l'équipement bio - 
psychologique de l'être humain, n'est en soi ni bonne, ni mauvaise ; seuls, certains de ses objets de satisfaction sont à proscrire.

\section{IV.2. L'énonciateur de l'interdit}

13 F. DOLTO, réfléchissant aux conditions nécessaires pour assurer la dimension symboligène du processus de castration, indique que celle - ci renvoie en définitive aux «qualités » de l'adulte mis en position de la donner : «Un enfant accepte une limitation et une temporisation à la satisfaction de ses désirs, et même un interdit de jamais les satisfaire, si la personne qui lui en fait l'interdit est une personne aimée, au pouvoir et au savoir de laquelle il sait qu'il est en droit d'accéder... Cet adulte ne peut faire accéder l'enfant à la symbolisation de ses pulsions que si... il est animé de respect et d'amour chaste pour l'enfant... » (p. 87). Ailleurs, le même auteur signale une autre condition, et non des moindres, à laquelle se doit d'être soumis l'énonciateur de l'interdit à un enfant : «La verbalisation de l'interdit donné à telle visée de son désir, à condition qu'il sache bien que l'adulte est aussi marqué que lui par cet interdit ${ }^{9}$, aide l'enfant à supporter l'épreuve, et la confiance demeure chez le sujet en son droit d'imaginer le but de ce désir que l'adulte a interdit. C'est donc par interdit que le sujet désirant est initié à la puissance de son désir, qui est une valeur, en même temps qu'il s'initie ainsi à la Loi, laquelle lui donne d'autres voies à l'identification des autres êtres humains, marqués, eux aussi, par la Loi » (p. 79).

Ce que cet auteur nous indique, en des termes remarquablement non techniques, en dépit de la densité de ses formulations, c'est que l'éducation, et spécialement ce moment éminent qui est l'énonciation de l'interdit, se déroulent dans un espace psychique qui peut être qualifié de «transféro - contra - transférentiel : l'efficace de cette parole qui fixe une limite et donne un interdit est, pour l'enfant, étroitement fonction de l'importance affective qu'il accorde à celui qui la profère, tout autant qu'elle dépend de la clarté - à entendre comme un processus progressif et permanent de clarification - de la position psychique de qui l'énonce ${ }^{10}$. Sur un tel terrain, nous ne manquons pas de rejoindre ce que l'on peut entendre, dans une perspective clinico - psychanalytique, par la notion d'autorité. Nous avons tenté, ailleurs, de la définir comme "L'efficace d'une parole, qui, au sein d'une relation transférentielle, touche au cœur le sujet, pour le faire grandir et le sortir de la confusion pulsionnelle dans laquelle il est initialement plongé $\aleph^{11}$

\section{IV.3. Le moment d'interdire...}

DOLTO nous introduit à une véritable intelligence dans le recours à l'usage de l'interdit. Il ne peut s'agir d'en user de manière anarchique, mais au contraire à bon escient, de telle sorte qu'il soit entendu de l'enfant à qui il s'adresse. Elle aimait illustrer ce point d'un exemple, évoquant la venue à la crèche d'un enfant qui se précipite dans la salle des jeux et observe d'emblée que le beau camion rouge avec lequel il désirait jouer, se trouve déjà accaparé par un copain, arrivé plus tôt! Notre sujet présente alors une réaction "pulsionnelle», s'approchant de l'autre la main levée, pour lui prendre l'objet de sa convoitise. A ce point précis de la séquence observée, DOLTO insiste bien sur la nécessité de l'intervention vigilante d'un adulte, personnel de la crèche, connu de l'enfant, et qui va interpeller celui - ci avec une fermeté telle qu'elle arrête l'action en cours. Et nous imaginons le petit, dans le suspens de son geste, tourné vers celui qui lui parle, attentif et qui s'entend dire : « Non, Kevin, tu n'as pas le droit de lui prendre, il l'avait avant toi. Tu 
peux soit attendre qu'il ait fini de jouer pour avoir le camion, soit lui demander de jouer avec lui...». Et DOLTO ne manquait jamais d'en conclure avec cet exemple par la question : « Comment apprenons-nous à nos enfants à demander plutôt qu'à prendre? ».

Mais surtout, la succession des interdits fondamentaux - les «castrations » - que tout enfant est en droit de recevoir, est déterminée par son organisation libidinale elle même. C'est la connaissance que nous en apporte la psychanalyse qui doit guider l'intervention de l'éducateur. DOLTO, ici, ne fait que suivre l'indication insistante de FREUD : l'éducation est l'un des domaines d'application de la psychanalyse. Ainsi peut-elle écrire : « Il y a le juste moment pour apporter chaque castration ; ce moment, c'est celui où déjà les pulsions, celles qui sont en cours, ont apporté un certain développement du schéma corporel qui rend l'enfant capable d'aménager ses plaisirs autrement que dans la satisfaction du total corps à corps, lequel n'est plus absolument nécessaire à ce spécimen de l'espèce humaine que représente l'organisme corps, pour qu'il survive en tant qu'être de besoin. Reste que, à cet organisme qui fait de l'enfant un être de besoin, est associé un sujet de désir » (p. 86).

\section{IV.4. La castration « ombilicale »}

17 C'est la première des "castrations " qu'identifie F. DOLTO; elle est constituée par la naissance et il peut paraître étonnant qu'elle ne s'assortisse pas d'un interdit explicite. Nous devons entendre en effet qu'il est question davantage d'un renoncement ${ }^{12}$, impliqué par la séparation, que d'une interdiction, tant pour le bébé que pour ses parents (ce que DOLTO appelle la castration «imaginaire » des parents) : «La cicatrice ombilicale et la perte du placenta peuvent, du fait de la suite du destin humain, être considérées comme une préfiguration de toutes les épreuves qu'on nommera plus tard castrations » (pp. 90 91). Mais, surtout, «C'est le langage qui symbolise la castration de la naissance ...» (p. 93) : l'audition de son prénom par le bébé, la nomination de son sexe vont de pair avec la perception des affects des parents, qui sont les traces symboligènes de la venue au monde. L'effet de la castration ombilicale, c'est de transférer la prévalence érogène de la région ombilicale vers la région orale.

\section{IV.5. La castration orale}

Elle signifie «La privation imposée au bébé de ce qui est pour lui le cannibalisme vis - à vis de sa mère : c'est - à - dire le sevrage, et aussi l'empêchement de consommer ce qui serait poison mortifère pour son corps, soit l'interdit de manger ce qui n'est pas alimentaire... Lorsqu'elle est judicieusement donnée, (elle) aboutit au désir et à la possibilité de parler, et donc à la découverte de nouveaux moyens de communication... » (p.99). DOLTO ne manque pas de rappeler combien le corps à corps entre mère et bébé est érotisant: la prise de distance, lorsqu'elle est intervenue à temps, ouvre dès lors « des possibilités de relation symbolique que cette castration a promues dans l'inconscient et dans le psychisme de l'enfant. » (p. 100). En des pages qui sont à la fois belles et précises, Françoise DOLTO décrit comment le bébé va peu à peu se désencombrer du sein maternel (ou du biberon), ainsi que du plaisir qui lui est attaché, pour entrer dans ce corps «subtil » qu'est le langage. 


\section{IV.6 La castration anale}

19

Dans la perspective de DOLTO, elle concerne moins la fonction excrémentielle que, plus généralement, le destin de la motricité volontaire, lorsque l'enfant y accède: « La castration anale, c'est l'interdit de nuire à son propre corps, comme au monde inanimé et animé qui entoure le triangle initial père - mère - enfant, par des agissements moteurs, rejetants, dangereux et non contrôlés. C'est ... l'interdit du meurtre et du vandalisme, au nom de la saine harmonie du groupe $»\left(\right.$ p. 108) ${ }^{13}$.

\section{IV.7 La castration « primaire »}

F. DOLTO la désigne encore comme « castration génitale non œdipienne » : elle concerne, à travers la découverte de la différence des sexes, l'accès aux débuts de l'identité sexuelle. Moments, on le sait, d'intenses curiosités, chez les garçons comme chez les filles, et d'intérêts très centrés. C'est alors que « des paroles vraies doivent être données à l'enfant des deux sexes, confirmant la justesse de son observation, et le félicitant de s'être aperçu d'une différence qui a toujours existé. Les paroles vraies qui réfêrent la conformité de son sexe $\grave{a}$ un avenir de femme ou d'homme, c'est cela qui donne valeur de langage et valeur sociale à son sexe et à lui- même » (p. 166) $)^{14}$.

\section{IV.8 La castration génitale œdipienne}

Elle concerne bien évidemment l'interdit de l'inceste, mais ce qui spécifie l'apport de Françoise DOLTO, c'est le caractère très concret et direct des formulations qu'elle suggère, de telle sorte que cet interdit ait une portée intrapsychique : «Quand cela est dit par le père à son fils, c'est l'initiation du fils à la vie humaine. C'est cela, la castration œdipienne. "Je t'interdis ta mère, parce qu'elle est ma femme, et qu'elle t'a mis au monde. Les deux sont importants. Tes sœurs te sont aussi interdites sexuellement que ta mère. Moi, je ne suis pas marié ni avec ma mère, ta grand - mère paternelle, ni avec tes tantes, qui sont mes sœurs ; ta mère ne s'est mariée ni avec son père, ton grand - père maternel, ni avec ses frères, etc. » (p. 190).

\section{Conclusion : Les effets de l'interdit, les «fruits » de la castration}

A partir du constat d'une progressive dévaluation de l'interdit dans la culture actuelle, et en particulier au sein des espaces éducatifs, nous nous sommes attachés à en comprendre la portée psychiquement structurante pour l'enfant. A cette fin, nous avons fait le détour par les travaux de Françoise DOLTO, dont l'un des apports centraux concerne précisément l'élucidation de la valeur et des effets des paroles vraies, apportées par l'adulte, à un enfant aux moments - clés de son développement psychique, ce qu'elle nomme dans sa terminologie propre des «castrations ». Leur incidence n'est pas que de surface; bien au contraire, elles vont contribuer à l'élaboration et à la structuration de l'image inconsciente du corps et à la promotion symboligène du sujet. 


\section{NOTES}

1. C'est nous qui soulignons.

2. «Lorsque, dans la composition de la classe, ce qui est le cas dans certains secteurs, le nombre d'élèves en opposition ou en situation de marginalité dépasse le seuil, ces élèves peuvent d'autant moins être contenus qu'ils sont soutenus, et même encouragés de l'extérieur par les grands - frères ou la bande. On passe alors à une classe - bataille très éprouvante pour les enseignants, le chef d'établissement et pour la majorité des élèves ». LEVINE 217.

3. LEGENDRE Pierre. 1998. L'inestimable objet de la transmission. Paris, Fayard.

4. FREUD Sigmund. 1932. Eclaircissements, applications, orientations (XXXIVe Leçon), in Nouvelles suites des leçons d'introduction à la psychanalyse, Euvres Complètes, XIX, pp. 233 - 234.

5. Une patiente, d'une cinquantaine d'années, m'a dit un jour, avec un inexprimable ressentiment dans le ton : " Moi, Monsieur, j'ai été élevée au garde - à - vous... ».

6. LACAN Jacques. 1938. Les complexes familiaux dans la formation de l'individu. Essai d'analyse d'une fonction en psychologie, in L'encyclopédie française, repris in (2001) Autres écrits, Paris, Seuil, pp. $60-61$.

7. LEVINE Jacques. 2003. Assistons - nous à la naissance d'un nouveau peuple scolaire ? Je est un autre, Bulletin de liaison de l'AGSAS, n 13, pp. 23 - 30. Rappelons que cet auteur apporte trois concepts, appareillés les uns aux autres, pour penser la situation scolaire actuelle: la déparentalisation, hypothèse d'une désolidarisation à l'égard des instances parentales ; l'auto parentalisation, mouvement par lequel le jeune s'adjuge un statut d'auto - procréateur ; et l'auto - suffisance générationnelle hors monde adulte, qui désigne l'illusion de former, entre jeunes, un monde quasi « autarcique ", en n'étant redevable de rien à personne ...

8. DOLTO Francoise. 1984. L'image inconsciente du corps, Paris, Seuil, p. 89 - 90.

9. C'est l'auteur qui souligne.

10. DOLTO observe également qu'il y a la possibilité «d'un effet symboligène pervers des castrations. Souvent tout - à - fait inconscient de la part des parents ou des éducateurs qui en sont à l'origine. Une castration qui induit le désir de se satisfaire dans la souffrance, au lieu de se satisfaire dans le plaisir, est une perversion. » (o.c. p 81). C'est l'auteur qui souligne.

11. GINET Dominique.2004. Aux racines de l'autorité, in CHAPPAZ Georges : L'autorité en panne ... Entre besoin de soumettre et désir d'éduquer. Actes de l'Université d'été 2003, Université de Provence, Marseille, p. 48.

12. Renoncement, en particulier, à «ce qui constituait in utero notre propre organisme, enveloppes amniotiques, placenta, cordon ombilical ; part grâce à laquelle nous avons pu être viables pour un autre espace qui, en nous accueillant, nous rend à jamais impossible le retour à l'espace précédent, au mode de vivre et de jouir que nous y avions connu ; (o.c. p. 92).

13. «La castration anale doit enseigner à l'enfant la différence entre ce qui est sa possession, dont il est totalement libre, et ce qui est la possession d'autrui, dont l'usage pour lui doit passer par la parole qui demande à autrui de lui prêter des objets dont il voudrait se servir, et qui accepte que cet autrui les lui refuse. » (p. 141).

14. C'est l'auteur qui souligne. 


\section{RÉSUMÉS}

A partir du constat du discrédit qui affecte aujourd'hui la notion d'interdit et qui semble désigner de façon symptomatique une lacune dans la transmission intergénérationnelle, l'auteur s'attache à revisiter, après ceux de FREUD et de LACAN, les apports spécifiques de Françoise DOLTO, en ce qu'ils permettent d'entendre, avec le concept de «castration symboligène », la portée psychiquement structurante des interdits.

Nowadays, the concept of the interdict is quite discredited. It seems to point out, in a symptomatic way, a gap in the inter - generation transmission. After studying FREUD's and LACAN's works, the author is now considering Françoise DOLTO's specific contributions, with regards to what the value of the concept of "castration sysmboligène " brings to the understanding of the structuring psychological impact of the interdicts.

\section{INDEX}

Mots-clés : autorité, interdit, castration, structuration psychique

Keywords : authority, interdict, castration, psychological structuration

\section{AUTEUR}

\section{DOMINIQUE GINET}

Maître de Conférences - Institut de Psychologie Groupe de recherche « Clinique du scolaire », Centre de Recherches sur les Inadaptations ; Université Lumière Lyon 2 